\title{
3D simulations of new low capacitance silicon pixel detectors
}

\author{
Qiang Long ${ }^{1, a}$, Qinwen Guo ${ }^{2, b}$, Zhongliang Long ${ }^{3, c}$ andZheng $\mathrm{Li}^{4, \mathrm{~d}}$ \\ ${ }^{1}$ School of Materials Science and Engineering, Xiangtan University, Xiangtan 411105, China \\ ${ }^{2}$ Center for Semiconductor Particle and photon Imaging Detector, Development and Fabrication, \\ Xiangtan University, Xiangtan 411105, China \\ ${ }^{3}$ National-Provincial Laboratory of Special Function Thin Film Materials, and School of Materials \\ alq1771297308@163.com, ’buoqinwen@hotmail.com, czhlong97@163.com, ${ }^{d}$ lizheng@xtu.edu.cn
}

Keywords: Low capacitance, signal to noise ratio, depletion voltage, the newly designed detector. Abstract. We propose a novel electrode structure that reduces the detector capacitance by employing a metal electrode with much reduced area as compared to the detector sensitive area. We found that by doing so, the full depletion voltage of the detector will increase due to the added lateral depletion between open areas of the same electrode. Therefore, this paper aims to optimize the performance of the detector by designing a reasonable electrode shape, weighing the relationship between capacitance and the required depletion voltage. Then, we compare the capacitance by simulating in detail the newly designed detector and compare with that in a traditional detector using a Silvaco TCAD simulation tool. And we show the depletion voltage, potential and electric field of two critical cross-sections of the newly designed detector.

\section{Introduction}

Nowadays, silicon detector, as a typical semiconductor detector, is widely used in medical, military and other fields due to it's excellent characteristics[1,2]. The silicon pixel detector is one of the new types of semiconductor silicon detector. Both Electrodes(cathodes and anodes) in conventional Si planar detectors are usually covered by a metal layer. This makes the detector capacitance proportional to the total area of the detector (which is defined by the metal area of the electrode). As the area of a detector increases, so does the detector capacitance and the associated electronic noise[3]. In order to increase the detector signal to noise ratio and improve the detector performance, one has to low the detector capacitance without decreasing the sensitive area.

The capacitance of a traditional pixel detector, with the signal electrode covering the entire detector area, is high. Low capacitance has been a critical requirement for designing a new detector. In this work, we propose a novel electrode structure that reduces the detector capacitance by employing a metal electrode with much reduced area as compared to the detector sensitive area. We also found that by doing so, the full depletion voltage of the detector will increase due to the added lateral depletion between open areas of the same electrode. In this paper, we mainly presented a kind of newly designed electrode shapes: The Closed Square Ring shape.

\section{Structure of new design pixel detector and planar detector}

We can simulate the Closed Square Ring shape and planar shape detector (the thickness is $503 \mu \mathrm{m}$ ) structure by using the Silvaco TCAD simulation tool. As shown in the Fig. 1, a and b are a single cell of the Closed Square Ring shape and planar shape respectively. At the bottom there is a layer of aluminum acts as the anode that is $1 \mu \mathrm{m}$ thick. Also, there is an $1 \mu \mathrm{m}$ thick $\mathrm{n}^{+}$-implanted layer (phosphorus, the peak concentration is $1 \times 10^{19} \mathrm{~cm}^{-3}$ ) on the top of the anode. And the bulk silicon is n-type high resistivity silicon doped with phosphorus of $8 \times 10^{11} \mathrm{~cm}^{-3}$. There are $1 \mu \mathrm{m}$ thick $\mathrm{p}^{+}-$ implanted areas (shown in Fig.1c)from $501 \mu \mathrm{m}$ to $502 \mu \mathrm{m}$. The difference between the Closed Square Ring shape and planar shape is that the cathodes of the former are separated by a silicon dioxide layer with an oxide charde density of $4 \times 10^{11} \mathrm{~cm}^{-3}$ when the cathodes is a square shape. In fact, the performance of the detector will be better with the decrease of the detector size[4,5]. In order to 
ensure that the heart shape designed sensor is fully exhausted under the voltage of $230 \mathrm{~V}$, we shall not allow the maximum spacing of the same electrode more than $233 \mu \mathrm{m}$. That is, the square edge of the center of the electrode shown in the image is less than $233 \mu \mathrm{m}$.

(a)
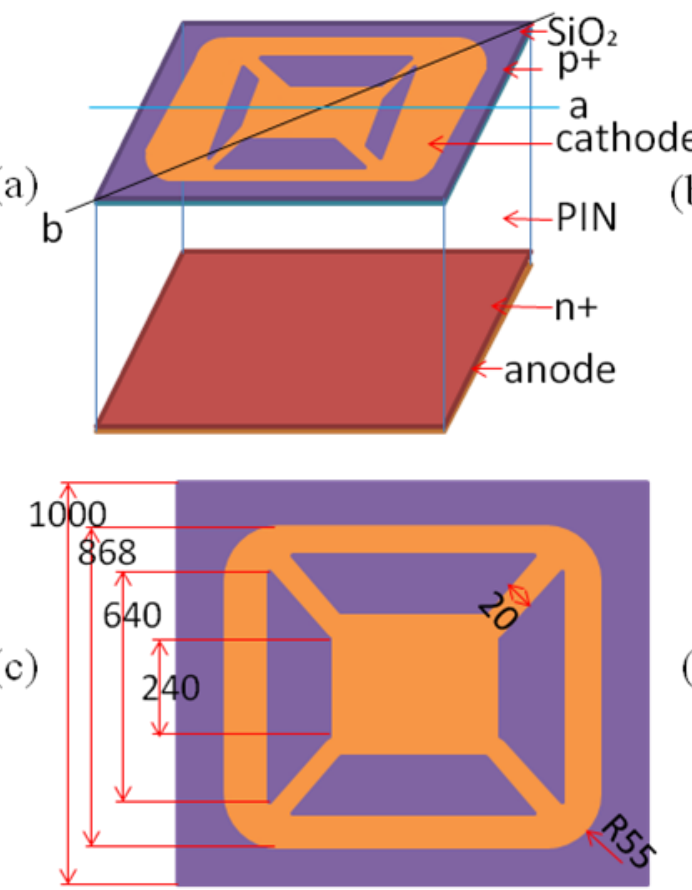

(b)
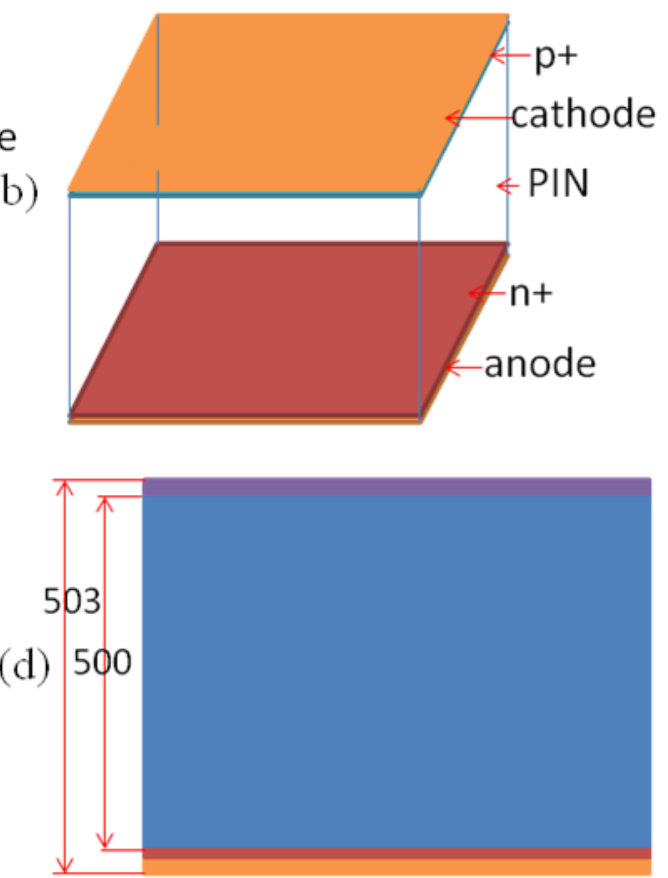

Fig.1.(a)Structure of the closed square ring shape detector. (b) Structure of the planar shape detector. (c) View of the probe structure of the closed square ring shape detector. (d) A view of the structure of the closed square ring shape detector a: cut plane-a.b:cut plane-b.
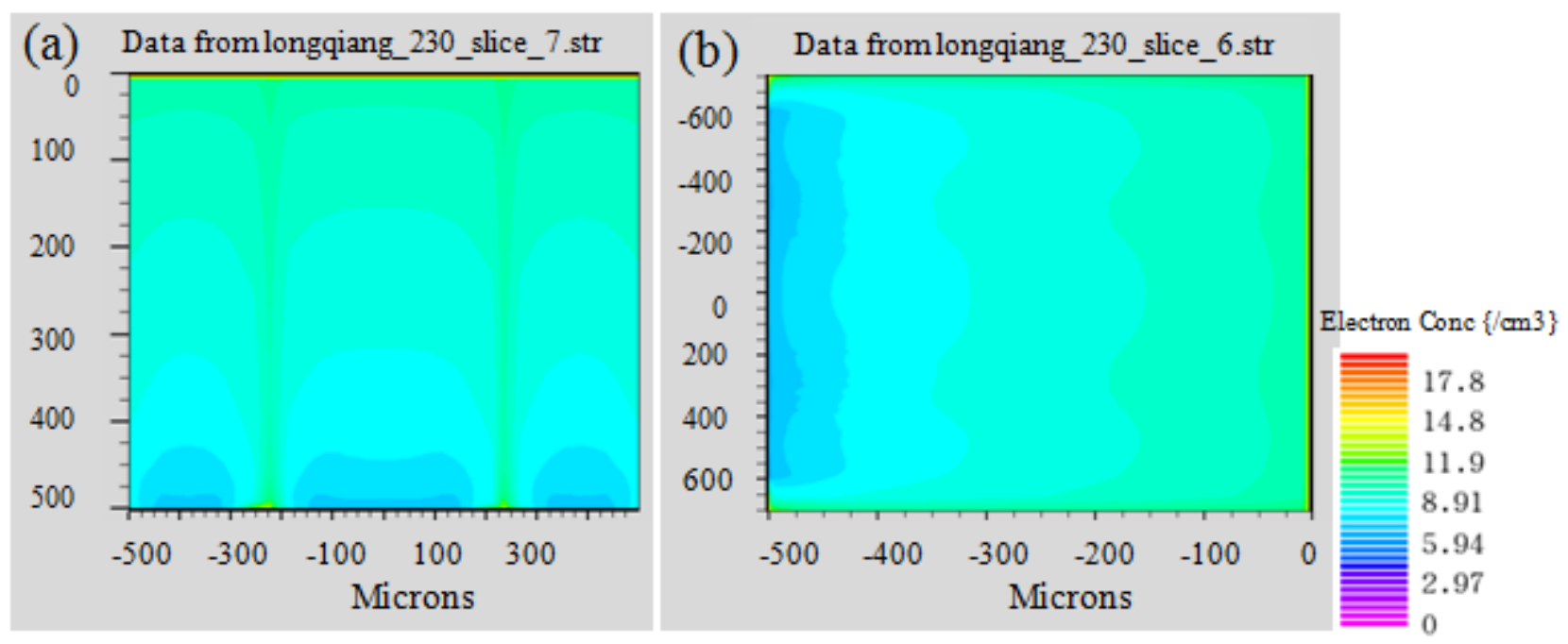

Fig.2. 2D electron concentration cut plane of the closed square ring shape detector at the full depletion voltage is about 230V. (a) Cross-section a. (b) Cross-section b.

\section{Full depletion voltage}

Shown in Fig. 2 is $2 \mathrm{D}$ cut plane (along the plane that has maximum distances between $\mathrm{p}^{+}$implants) electron concentration profiles of the two cross-section of the structure of the closed square ring shape detector. It can be seen from figure 2 that at the voltage of $230 \mathrm{~V}$, both sections are completely exhausted, and these two surfaces are important sections. So the detector can run out at the voltage of $230 \mathrm{~V}$. 


\section{Electric potential characteristics}

Fig. 3 shows the electric potential change of two critical cross-sections of the new detector at a bias voltage of 230 V. Fig. 3 (a) and (b) are the y-z slices of the face of the detectors and sectional drawing (see Fig. 1 (a)).
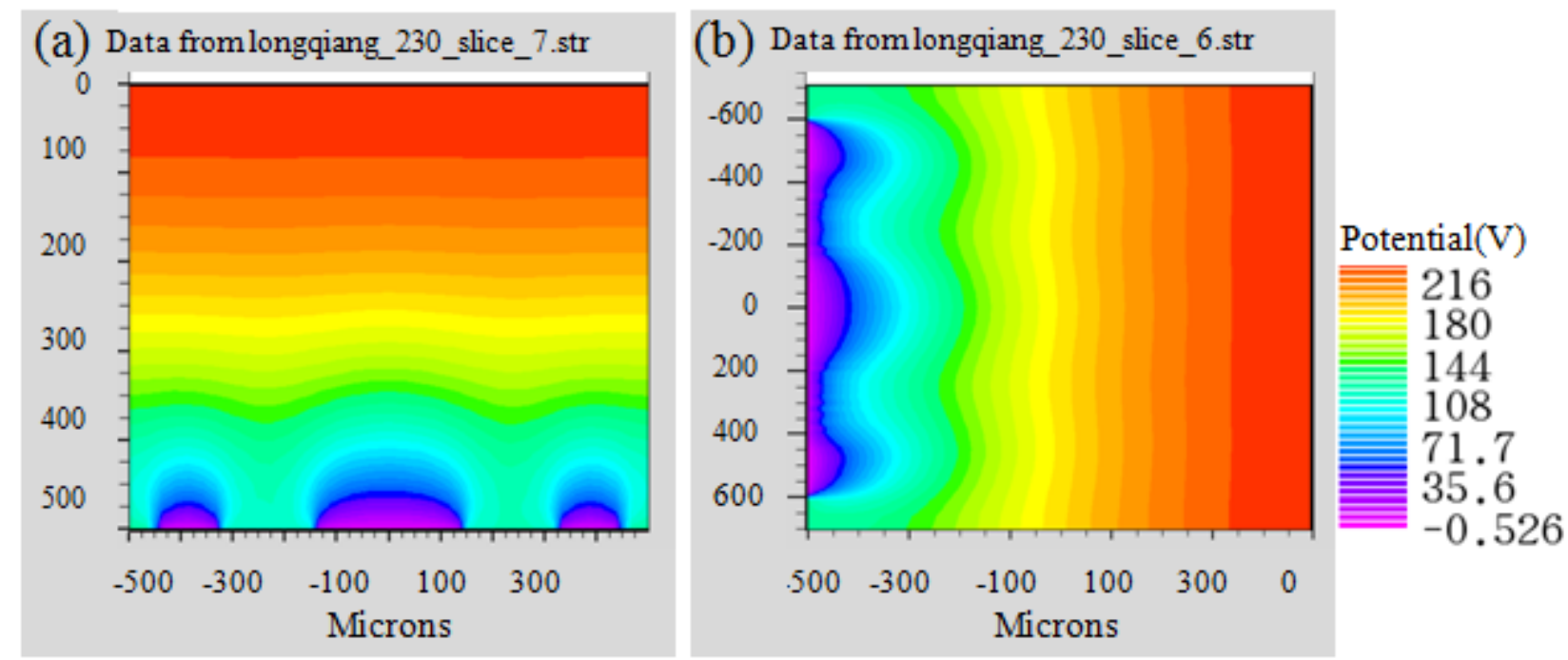

Fig.3. Potential profile of the closed square ring detector. (a)Cross-section a.

(b) Cross-section b.

\section{Capacitance-voltage characteristics}

The capacitance-voltage characteristics are obtained by using a small signal analysis (AC analysis, frequency is $1 \times 10^{6} \mathrm{~Hz}$ )[6]. The capacitance is a sensitive parameter in the operation of a silicon tracking detector, as it directly affects the noise and crosstalk[6]. The capacitance of the conventional detector is about $207 \mathrm{fF}$, but the capacitance of the new detector is $200 \mathrm{fF}$. It's about a thirtieth down.

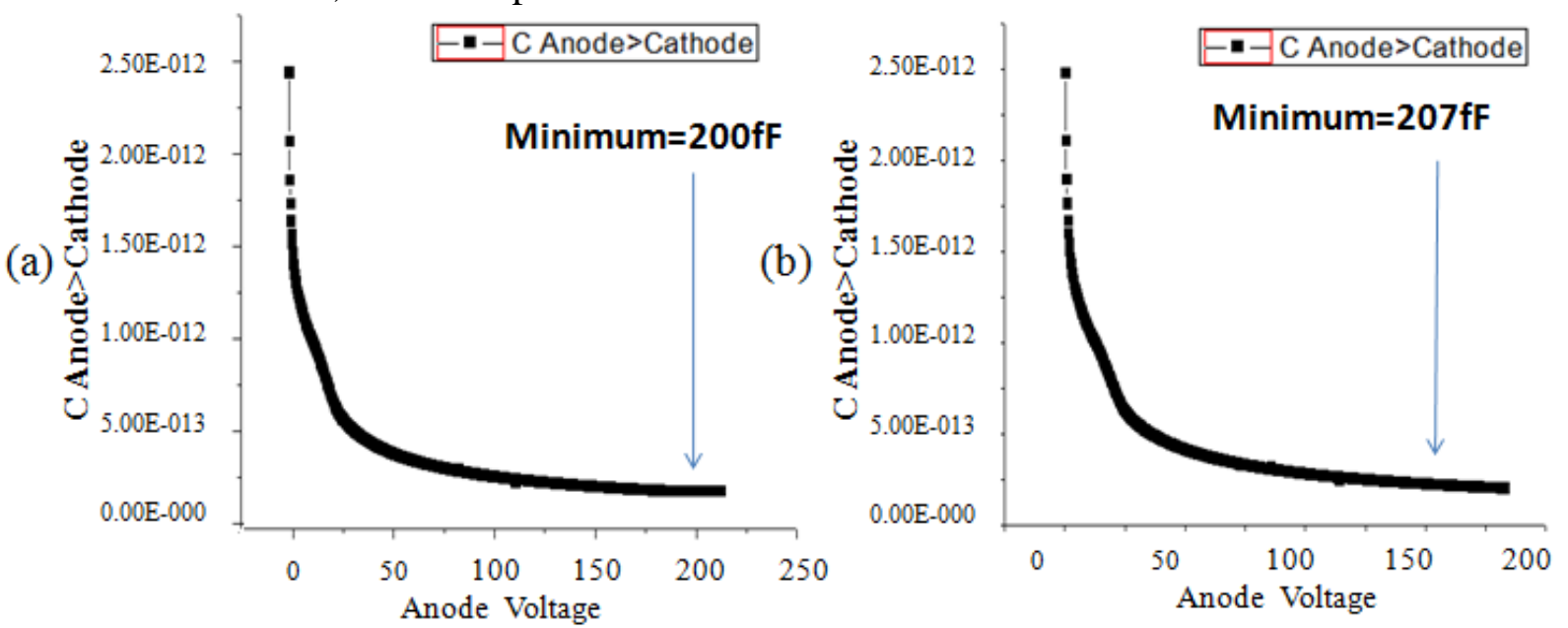

Fig.4. (a) The capacitance of the closed square ring shape detector(200fF).

(b) The capacitance of the planar shape detector(207fF). 


\section{Electric field characteristics}

Fig. 5 shows the electric field change of two critical cross-sections of the new detector at a bias voltage of 230 V. Fig. 5 (a) and (b) are the y-z slices of the face of the detectors and sectional drawing (see Fig. 1 (a)).
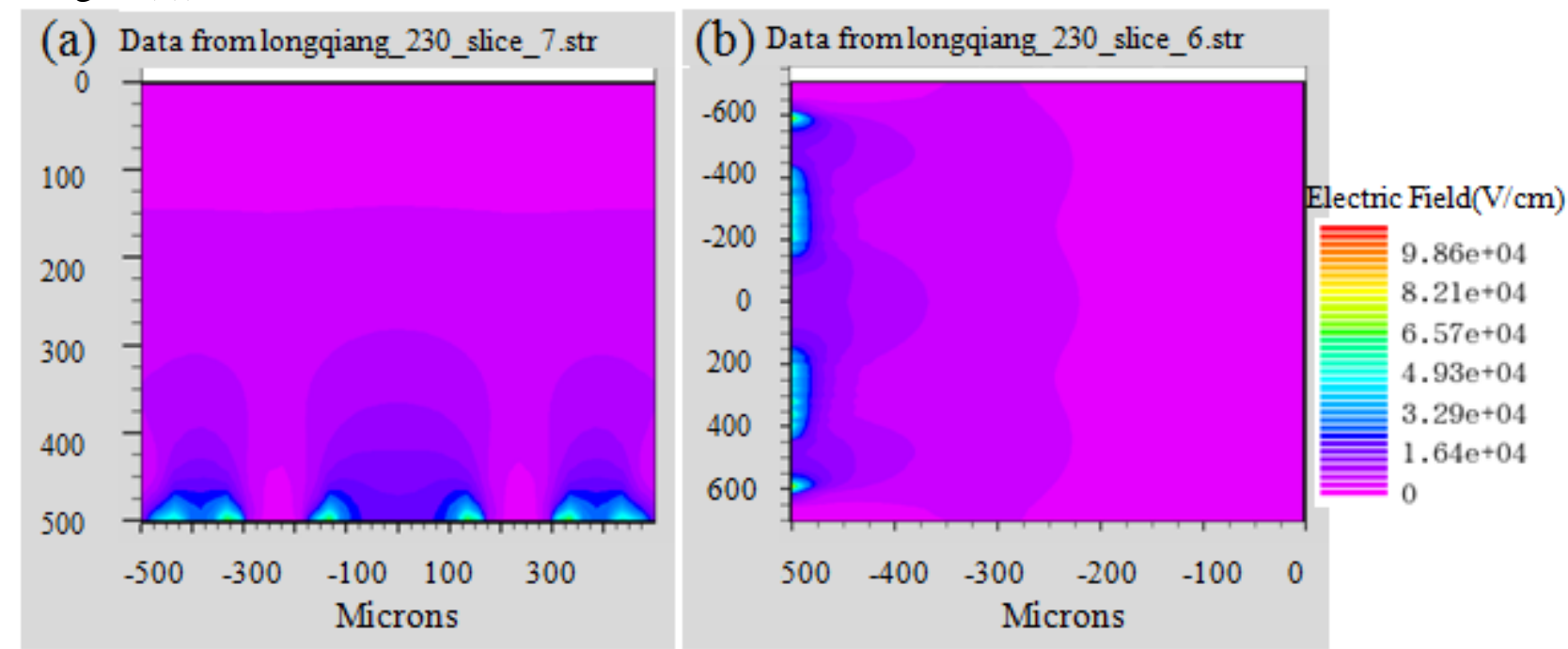

Fig.5.Electric field profile of the closed square ring detector.(a)Crosssection a.(b)Cross-section b.

\section{Conclusion}

We have designed a new type of silicon pixel detector, and we can know that capacitance of the new detector is reduced by a thirtieth compared with the traditional detector by three-dimensional simulation calculation. Reducing the area of the electrode can reduce the capacitance and increase the depletion voltage required by the detector. We have designed a new detector that balances the two. The capacitance is reduced effectively and the detector can run out at a certain voltage. In fact, we designed the detector to run out at $230 \mathrm{~V}$. And it fits well with the performance requirements we need.

\section{References}

[1] Z. Gianluigi De Geronimo. D. Li, Peter Siddons, et al., IEEE Transactions on Nuclear Science NS57 (3) (2010) 1654.

[2] D.P. Siddons, A. Dragone, G. De Geronimo, Z. Li, et al.IEEE Nuclear Science Symposium Conference Record. Nov, 725-727,2005.

[3] Z. Li, Nuclear Instruments and Methods Section A 612 (2010) 509.

[4] Z. Li, N uclear Instruments and Methods Section A 518 (2004) 738.

[5] Z. Li, W. Chen, Y.H. Guo. Nuclear Instruments and Methods Sention A 538 (2007) 139.

[6] J. Chen, et al., Nuclear Instruments and Methods Section A 796 (2015) 34.

[7] G. Gorfine, et al., Nuclear Instruments and Methods Section A 465 (2001) 70. 